\title{
Asymptotic Safety and Lattice Quantum Gravity
}

\section{Jack Laiho*}

SUPA, School of Physics and Astronomy, University of Glasgow, Glasgow, G12 8QQ, UK

E-mail: jlaiho@physics.gla.ac.uk

\section{Daniel Coumbe}

SUPA, School of Physics and Astronomy, University of Glasgow, Glasgow, G12 8QQ, UK

E-mail: d.coumbe@physics.gla.ac.uk

We review evidence presented recently that a nonperturbative formulation of quantum gravity defined by Euclidean Dynamical Triangulations with a nontrivial measure term gives rise to a phase with extended, four-dimensional geometries. The results are strikingly consistent with those of Causal Dynamical Triangulations for both the Hausdorff dimension, which is consistent with 4 , and for the spectral dimension, which exhibits a scale dependence, running to 4 at long distances and to smaller values at short distances. We discuss some of the implications that this has for the asymptotic safety scenario for quantum gravity.

The XXIX International Symposium on Lattice Field Theory - Lattice 2011

July 10-16, 2011

Squaw Valley, Lake Tahoe, California

\footnotetext{
*Speaker.
} 


\section{Introduction and Motivation}

The formulation of a consistent, predictive quantum theory of gravity is one of the great outstanding problems in theoretical physics. The most straightforward implementation of general relativity as a quantum field theory is nonrenormalizable when formulated perturbatively [1]. Although the theory can be interpreted as an effective field theory at energies well below the Planck scale, as one goes to higher loop order in the effective theory, new divergences appear, requiring an infinite number of couplings that parameterize the physics at high energy scales [2]. This leads to a loss of predictive power.

Weinberg suggested decades ago that the effective field theory of gravity might be asymptotically safe [3], i.e. that gravity might be effectively renormalizable when formulated nonperturbatively. In this scenario, the renormalization group flows of couplings have a non-trivial fixed point, with a finite dimensional ultraviolet critical surface of trajectories attracted to a fixed point at short distances. In a lattice formulation, the fixed point would appear as a second order critical point, the approach to which would define a continuum limit. The divergent correlation length characteristic of a second order phase transition would allow one to take the lattice spacing to zero while keeping observable quantities fixed in physical units. Asymptotically free theories (such as QCD) are a special case of asymptotically safe theories, where the fixed point is the Gaussian fixed point. Such theories become noninteracting in the high energy limit. For gravity, because the theory is perturbatively nonrenormalizable, the theory is not asymptotically free, but may still possess a nontrivial fixed point, so that it is asymptotically safe. The investigation of the nontrivial fixed point appearing in such a theory requires nonperturbative methods. ${ }^{1}$

There has been a significant amount of work done on the asymptotic safety scenario of gravity using continuum functional renormalization group methods $[5,6,7,8,9,10]$, as well as lattice calculations $[29,12,13,14]$. The renormalization group methods have shown the existence of a nontrivial fixed point in a variety of truncations of the renormalization group equations, indicating that the dimension of the ultraviolet critical surface is finite. Various truncations with more than three independent couplings suggest that the critical surface is three dimensional. When the number of couplings in various truncations is increased beyond three, the dimension of the ultraviolet critical surface remains three $[8,10]$.

The renormalization group studies are suggestive, but these studies have been done with a truncation of the (infinite) number of terms in the effective action, so that it is difficult to systematically assess the reliability of the results using this method. In particular, is the presence of the fixed point an artifact of the truncation, or a property of the full, untruncated effective action? The persistence of the fixed point for a wide variety of truncations suggests that the fixed point is a property of the full theory, but assessing the reliability of this conclusion is difficult when we do not have a controlled expansion to constrain the size of the contribution from operators that are left out. Given what is at stake, a lattice formulation is desirable, since this in principle allows one to

\footnotetext{
${ }^{1}$ If one includes higher derivative terms in the gravitational action and performs a resummation such that the higher derivative terms are incorporated into the graviton propagator, then gravity is renormalizable by power counting and asymptotically free, but has problems with unitarity at high energy scales due to the presence of ghosts [4]. However, the ghost poles are of order the Planck mass, so the unitarity violations are only significant in the high-energy regime where perturbation theory breaks down anyway, and this further points to the need for nonperturbative methods.
} 
perform calculations with controlled systematic errors. It is important to consider what the lattice has to say about asymptotically safe gravity because different theoretical methods are needed to cross-check each other and to give some indication of whether we are on the right track.

\section{Dynamical Triangulations}

Euclidean Dynamical Triangulations (EDT) is a variant of quantum Regge calculus $[15,16]$, where the lattice geometries entering the Euclidean path integral are approximated by gluing together the higher dimensional analogs of equilateral triangles, keeping the edge lengths fixed. These building blocks are called simplices, and we will restrict ourselves in this proceeding to the case where the simplices being glued together are four-dimensional. Since the edge lengths are fixed, the dynamics is encoded in the connectivity of the four-simplices. Although the dimension of the simplices is fixed, the effective dimension of the geometries at scales large compared to the lattice spacing will not necessarily be the same as that of the building blocks. Earlier lattice calculations from the '90's showed that there were two phases in the simplest implementation of the model, though neither of these phases looked much like semiclassical general relativity in four dimensions [15, 19, 20, 21, 22]. Furthermore, the critical point separating these two phases, though initially thought to be second order, turned out to be first-order, thus ruling out the usual approach of taking a continuum limit $[17,18]$. This result was rather discouraging, and indeed, work on Euclidean Dynamical Triangulations largely died out after this result was found.

A new approach based on the original dynamical triangulations model was introduced by Ambjorn and Loll [23], mainly in response to these difficulties. This new approach is called Causal Dynamical Triangulations (CDT) because of the causality condition that is imposed on the triangulations over which the path integral sum is taken. In CDT, one distinguishes between spacelike and timelike links on the lattice, so that an explicit foliation of the lattice into spacelike hypersurfaces can be introduced. The constraint requires that the hypersurfaces have the same topology, usually chosen to be $S^{3}$. This prevents branching of the geometries into "baby universes" along the time direction. In CDT, only geometries that admit such a global foliation are included in the space of triangulations defining the measure of the path integral [24].

The CDT calculations show that a phase with a good semiclassical limit exists within the parameter space of couplings that define the model $[25,26]$. Within this phase there emerges a ground state consistent with four dimensional de Sitter space [26], including quantum fluctuations that are well described by a semiclassical expansion about de Sitter space [12,13]. There is also strong evidence for the existence of a second order critical line in the phase diagram at one of the boundaries of the de Sitter-like phase; points on this line are thus candidates for non-trivial fixed points [27]. Additionally, it was shown that the spectral dimension (a type of fractal dimension defined by a diffusion process) varies as a function of distance $[28,29]$. The different definitions of fractal dimensions are in general different on a fractal geometry, but they reduce to the usual definition of dimension on smooth space-times. The spectral dimension of CDT is found to be close to four at large distances, as one would expect for a theory of gravity describing our world at long distances. At short distances the spectral dimension decreases to smaller values, in accord with renormalization group calculations that support the asymptotic safety scenario [30], as well as other approaches to quantum gravity [32,31]. It is not yet known if CDT is in the same universality 
class as any of these other theories, or whether more than one universality class can be reached from different critical points in the phase diagram of CDT. For a review of CDT aimed at a lattice audience, see last year's conference proceedings by Renate Loll [33].

\section{Revisiting EDT}

Based on the successes of CDT, there are a number of reasons for revisiting the EDT formulation. One of these is that fixing the foliation in CDT is potentially at odds with general covariance. If, however, the foliation amounts to a gauge-fixing procedure, then it should be possible to obtain the same results using an EDT formulation, which is explicitly covariant from the outset.

Another one of the original motivations for revisiting EDT was the following: there are three parameters that enter the bare lattice action in CDT, whereas the original EDT studies typically included only two. If we take seriously the renormalization group results suggesting that the ultraviolet critical surface is three dimensional, then we may need to include a third parameter in the bare action. Both EDT and CDT include the bare Newton and cosmological constants. CDT also introduces an anisotropy ratio of the lengths of timelike and spacelike links, so that CDT geometries are anisotropic along the time direction [34]. Thus, we consider the EDT formulation with a third parameter.

Our work of Ref. [35] is reviewed here; it involves calculations with EDT including a nontrivial measure term [36] and an accompanying free parameter. The path integral for Euclidean gravity has the partition function $[15,37]$

$$
Z_{E}=\sum_{T} \frac{1}{C_{T}}\left[\prod_{j=1}^{N_{2}} o\left(t_{j}\right)^{\beta}\right] e^{-S_{E}}
$$

where $C_{T}$ is a symmetry factor that divides out the number of equivalent ways of labeling the vertices in the triangulation $T$. The Euclidean Einstein-Regge action is

$$
S_{E}=-\kappa_{2} N_{2}+\kappa_{4} N_{4}
$$

where $N_{i}$ is the number of simplices of dimension $i$, and $\kappa_{2}$ and $\kappa_{4}$ can be related to the bare Newton's constant $G_{N}$ and the bare cosmological constant $\Lambda$. The term in brackets in Eq. (3.1) is the nontrivial measure term, where the product is over all two-simplices (triangles), and $o\left(t_{j}\right)$ is the order of triangle $j$, i.e. the number of four-simplices to which the triangle belongs. This corresponds in the continuum to a nonuniform weighting of the measure by $[\operatorname{det}(-g)]^{\beta / 2}$. We vary $\beta$ as an additional independent parameter in the action (after exponentiating the measure term). Most of the previous work on EDT considered the partition function in Eq. (3.1) with $\beta=0$ only.

The numerical implementation is by now well-established [38]. We use the set of local (p,q) moves [16, 39] with the Metropolis algorithm to perform the Monte Carlo integration, and we tune the bare cosmological constant (or equivalently $\kappa_{4}$ ) to its critical value in order to take the infinite volume limit. We also work at (nearly) fixed four-volume by including a term in the action $\delta \lambda\left|N_{4}^{f}-N_{4}\right|$ to keep the four-volume close to the value $N_{4}^{f}$. This does not alter the action at values of $N_{4}=N_{4}^{f}$, but serves to keep the volume fluctuations about $N_{4}^{f}$ from growing too large to be practical to simulate. Most of the early work on EDT used geometries constructed of combinatorial 


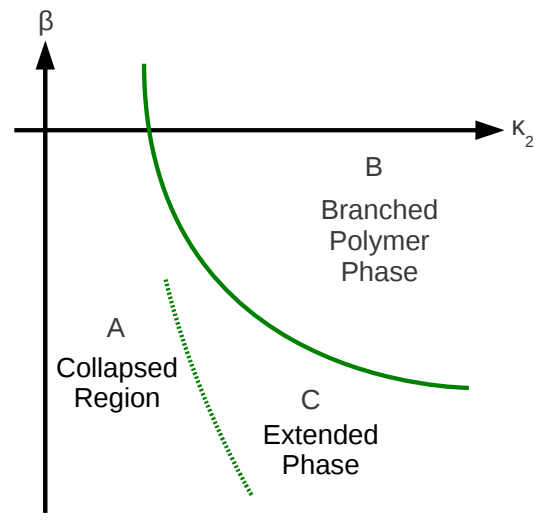

Figure 1: Schematic of the phase diagram as a function of $\kappa_{2}$ and $\beta$.

triangulations, where each 4-simplex has a simplex label and a set of combinatorially unique vertex labels. However, it was shown that combinatorial uniqueness can be relaxed to include a larger set of degenerate triangulations in which the neighbors of a given simplex are no longer unique [40]. It has also been shown numerically that simulations using degenerate triangulations lead to a factor of $\sim 10$ reduction in finite size effects compare to combinatorial triangulations [40]. For this reason, we adopt degenerate triangulations in our own calculations.

We do not yet know whether the exponent in the measure term is truly a relevant parameter, or whether the nontrivial measure with some value of $\beta$ is required as part of a consistent regularization using the method of Euclidean dynamical triangulations. This is currently under investigation. As we discuss below, once we include the measure term in the EDT simulations we find a wellbehaved, four-dimensional, semiclassical phase with properties that are strikingly similar to those of the semiclassical phase of CDT.

\section{The Phase Diagram of EDT}

We have revisited the phase diagram of EDT with a nontrivial measure term [41]. A schematic of the phase diagram is shown in Fig. 1 . Since $\kappa_{4}$ is adjusted to take the infinite volume limit, this leaves a two dimensional parameter space, which is explored by varying $\kappa_{2}$ and $\beta$. We use the average curvature of the geometries as an order parameter, as described in another entry of these proceedings. The line at $\beta=0$ has received much attention in previous studies, with a first-order phase transition separating two phases, one a branched polymer phase, and the other a collapsed phase. Neither of these phases much resembles semiclassical gravity [15, 19, 20, 21, 22]. The branched polymer phase has a Hausdorff dimension of two, while the collapsed phase has a very large and possibly infinite Hausdorff dimension. The Hausdorff dimension is a particular definition of fractal dimension, and differs from other definitions of fractal dimension, such as the spectral dimension, which is defined via a diffusion process. The spectral dimension of the branched polymer phase is $4 / 3$, a result that is known analytically [42] and is confirmed by numerical calculations. 


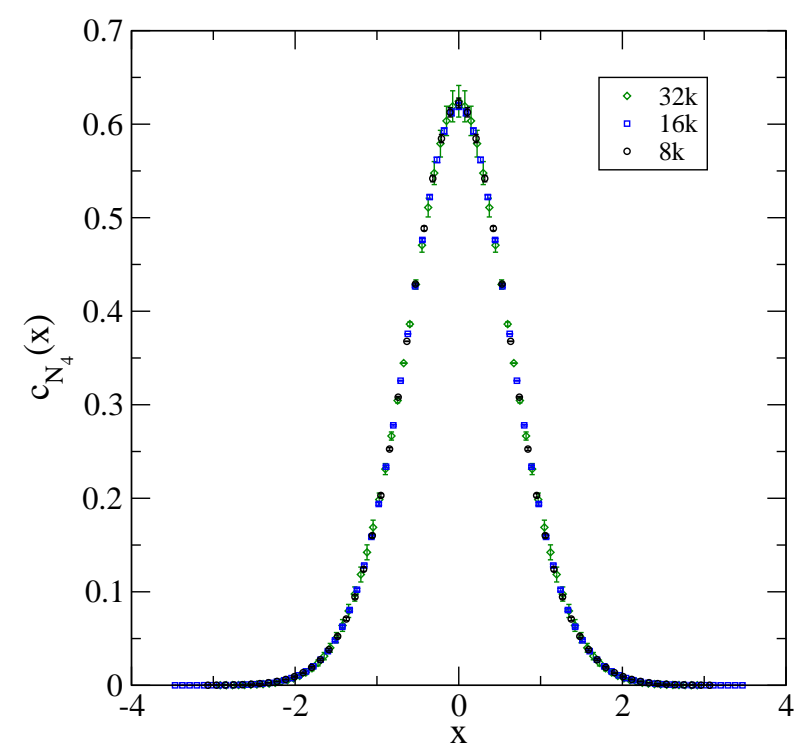

Figure 2: Three-volume correlator as a function of rescaled Euclidean time $x$ for three different volumes, $N_{4}=8000,16000$, and 32000 .

We find that there is a region of the phase diagram where the geometries are four-dimensional and de Sitter-like; we call this region the extended phase after a similar phase discovered in the CDT simulations [29]. Earlier studies of EDT were performed with the same nontrivial measure we are using, and these studies identified a qualitatively different type of geometry for sufficiently negative $\beta$, a region in the phase diagram that was dubbed the "crinkled" phase [37]. Based on our studies and a comparison with the CDT results, we believe that the name extended phase is more appropriate. The transition from the extended phase to the collapsed phase appears to be a cross-over, rather than a clear phase transition, suggesting that the two are not distinct phases, but that what has been called the collapsed phase is actually a region of the extended phase with particularly large discretization effects. Finer lattices can be reached by taking $\kappa_{2}$ large within the extended phase. We also find evidence for de Sitter behavior in the extended phase of EDT [43].

\section{Determining fractal dimensions}

We discuss here our determinations of the Hausdorff dimension and the spectral dimension in the extended phase. The Hausdorff dimension was determined using the finite volume scaling of a three-volume correlator similar to the one introduced in Ref. [29] to study the scaling of CDT,

$$
C_{N_{4}}(\delta)=\sum_{\tau=1}^{t} \frac{\left\langle N_{4}^{\text {slice }}(\tau) N_{4}^{\text {slice }}(t+\delta)\right\rangle}{N_{4}^{2}}
$$


where $N_{4}^{\text {slice }}(\tau)$ is the total number of 4-simplices in a spherical shell a geodesic distance $\tau$ from a randomly chosen simplex. $N_{4}$ is the total number of 4-simplices and the normalization of the correlator is chosen such that $\sum_{\delta=0}^{t-1} C_{N_{4}}(\delta)=1$. If we rescale $\delta$ and $C_{N_{4}}(\delta)$, defining $x=\delta / N_{4}^{1 / D_{H}}$, $c_{N_{4}}(x)=N_{4}^{1 / D_{H}} C_{N_{4}}\left(\delta / N_{4}^{1 / D_{H}}\right)$, then $c_{N_{4}}(x)$ should be independent of the lattice volume. One can determine the Hausdorff dimension $D_{H}$ from the value that leaves $c_{N_{4}}(x)$ invariant under a change in four-volume $N_{4}$. Good agreement between $c_{N_{4}}(x)$ at different volumes occurs when $D_{H}=4$, as can be seen in Fig. 2. Thus, the Hausdorff dimension is close to 4 in our extended phase. Evidence for the de Sitter nature of this phase will be presented elsewhere [43].

The spectral dimension of the lattice geometries in the extended phase is obtained using

$$
D_{S}(\sigma) \approx-2 \frac{d \log \langle P(\sigma)\rangle}{d \log \sigma}
$$

where $\langle P(\sigma)\rangle$ is the ensemble average of the return probability, i.e. the probability that a random walk will return to its origin after $\sigma$ steps. This formula receives finite-size corrections when $\sigma$ becomes much larger than the lattice volume $N_{4}^{2 / D_{S}}$. Figure 3 shows our results for $D_{S}$ as a function of $\sigma$, including a fit to the function

$$
D_{S}(\sigma)=a-\frac{b}{c+\sigma}
$$

suggested in Ref [29]. Our preferred fit to the spectral dimension gives $D_{S}(\sigma \rightarrow \infty)=4.04 \pm 0.26$ and $D_{S}(\sigma \rightarrow 0)=1.457 \pm 0.064$, where the error includes the statistical error and a systematic error associated with varying the fitting range and the fit function added to the statistical error in quadrature [35]. Variations in the fit function assume that the spectral dimension approaches a constant value in the $\sigma \rightarrow \infty$ limit, and that $D_{S}(\sigma)$ is monotonic. Although this result for the spectral dimension contains an estimate of the systematic error, to bring this error fully under control we need to quantify the finite size effects for $D_{S}(\sigma \rightarrow \infty)$ and discretization effects for $D_{S}(\sigma \rightarrow 0)$. Work in progress using ensembles with larger volumes and longer diffusion times still give $D_{S}(\sigma \rightarrow \infty)$ consistent with four. So far, our results at finer lattice spacings are also still consistent with $D_{S}(0)=3 / 2$, where the relative lattice spacing is determined from a comparison of the running spectral dimension at different values of the bare parameters.

Our results for the Hausdorff and spectral dimensions are consistent with the earlier studies of Ref. [37], but their identification of what we call the extended phase as "crinkled" suggests unphysical characteristics. One of the unphysical features attributed to the "crinkled" phase in Ref. [37] was the value of the spectral dimension $\sim 1.5-1.7$, results that are consistent with ours only at short distances. For simulations that we carried out at exactly the parameters chosen in Ref. [37], we find good agreement if we restrict ourselves to short diffusion times and ignore any variation of $D_{S}$ with $\sigma$. However, since we find that the spectral dimension in the extended phase depends on the distance scale probed, we see that this can still be consistent with $D_{S}$ running to a value close to 4 for sufficiently large scales, similar to what is seen in CDT simulations.

\section{Asymptotic Safety and Holography}

The similar results from EDT and CDT point to the possibility that the formulations may be in the same universality class, with a nontrivial fixed point governing the universal behavior. Could 


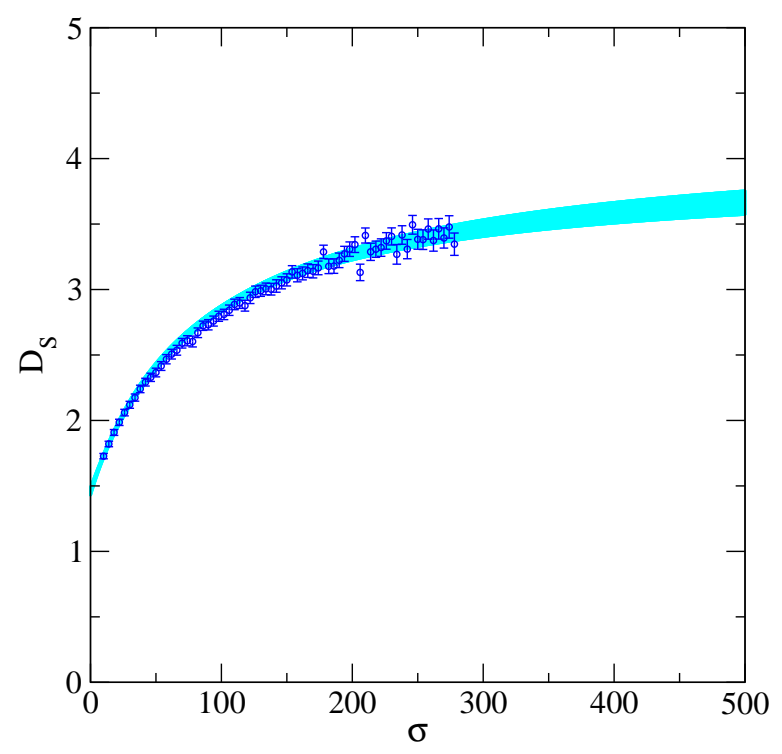

Figure 3: Spectral dimension as a function of diffusion time $\sigma$, including a fit to Eq.(5.3). The width of the band shows the statistical error in the fit.

asymptotic safety be correct? There is an argument against the possibility of the asymptotic safety scenario due to Banks [44] (see also Shomer [45]). The argument compares the density of states at high energies expected for a theory of gravity to that of a conformal field theory. Since a renormalizable quantum field theory is a perturbation of a conformal field theory by relevant operators, a renormalizable field theory must have the same high energy asymptotic density of states as a conformal field theory. It follows from dimensional analysis, the extensive scaling of energy and entropy, and the fact that a finite-temperature conformal field theory has no dimensionful scales other than the temperature, that the entropy $S$ and energy $E$ scale as

$$
S \sim(R T)^{d-1}, \quad E \sim R^{d-1} T^{d}
$$

where $R$ is the radius of space-time under consideration, and $T$ is the temperature. It follows that the entropy of a renormalizable theory must scale with energy as

$$
S \sim E^{\frac{d-1}{d}} .
$$

For gravity, however, one expects that the high energy spectrum will be dominated by black holes. The $d$ dimensional Schwarzschild solution in asymptotically flat space-time has a black hole with event horizon of radius $r^{d-3} \sim G_{N} M$, where $G_{N}$ is Newton's constant and $M$ is the mass of the black hole. The Bekenstein-Hawking entropy formula tells us that $S \sim r^{d-2}$, so that

$$
S \sim E^{\frac{d-2}{d-3}} .
$$

This scaling disagrees with that of Eq. (6.2). Thus, one is led to conclude that gravity cannot be formulated as a renormalizable quantum field theory. This is a potentially serious obstacle for 
asymptotic safety, though whether the semiclassical arguments on which it is based survive a full quantum treatment have been questioned [46].

We note here a possible resolution of this conflict. The correct dimension to use for the asymptotic density of states in Eq. (6.2) is the spectral dimension at short distance scales [47]. Under the plausible assumption that the relevant dimension in the holographic scaling argument is also the spectral dimension, we see that Eqs. (6.2) and (6.3) agree precisely when $D_{S}=3 / 2$. Then our EDT result for $D_{S}(0)$ resolves the tension between asymptotic safety and holographic entropy scaling. It is interesting to note that the CDT result $D_{S}(0)=1.80 \pm 0.25$ [29] is not inconsistent with $3 / 2$, and further precision here would be desirable, especially given the renormalization group argument that $D_{S}(0)=2$ exactly $[30]^{2}$. The renormalization group results and our lattice results may not be inconsistent if the behavior of $D_{S}(\sigma)$ for small $\sigma$ is non-monotonic, as discussed in Ref. [49]. Finer lattice spacings are most likely required to see whether the non-monotonic behavior proposed in Ref. [49] to explain the discrepancy between the lattice and the renormalization group results for $D_{S}(0)$ is realized.

\section{Conclusions and Outlook}

The existence of a four dimensional extended phase in the EDT model is a promising sign for lattice attempts to realize the asymptotic safety scenario. The results for the running spectral dimension provide nontrivial evidence that CDT and EDT may be in the same universality class. If so, the two approaches would be complementary. In CDT there exists a reflection-positive transfer matrix [24,50], pointing to the theory being unitary, whereas the EDT formulation maintains a fully symmetric treatment of space and time throughout, in the spirit of general covariance. Whether or not other approaches, such as Regge Calculus [51], or gauge formulations of gravity [52], lead to the same universality class should also be explored. If a continuum limit at a second order critical point exists, and EDT and CDT are in the same universality class, this would point to the existence of a unitary, covariant, renormalizable quantum field theory of general relativity. The discussion of the previous section indicates how such a theory may yet be consistent with black hole entropy scaling.

If this scenario holds up, there will be many things to look at next. Adding matter to the calculations is an obvious next step. Indeed, there are many questions one can ask. Is it possible to make contact with observation, in particular with cosmology and the early universe? Does an asymptotically safe theory of gravity with a suitable matter sector lead to predictions for low energy parameters [53], and can the lattice help sharpen such predictions? Can the lattice make contact with other approaches to quantum gravity? What happens when we add more dimensions? I am hopeful that future lattice calculations will be able to help answer these questions.

\section{References}

[1] M. H. Goroff and A. Sagnotti, Nucl. Phys. B 266, 709 (1986).

[2] J. Donoghue, gr-qc/9712070.

\footnotetext{
${ }^{2}$ See Ref. [48] for a possible loop-hole in the renormalization group argument.
} 
[3] S. Weinberg, In *Hawking, S.W., Israel, W.: General Relativity*, 790-831 (1978).

[4] K. S. Stelle, Phys. Rev. D 16, 953 (1977).

[5] O. Lauscher and M. Reuter, Phys. Rev. D 65, 025013 (2002) [hep-th/0108040].

[6] O. Lauscher and M. Reuter, Class. Quant. Grav. 19, 483 (2002) [hep-th/0110021].

[7] D. F. Litim, Phys. Rev. Lett. 92, 201301 (2004) [hep-th/0312114].

[8] A. Codello, R. Percacci and C. Rahmede, Int. J. Mod. Phys. A 23, 143 (2008) [arXiv:0705.1769 [hep-th]].

[9] A. Codello, R. Percacci and C. Rahmede, Annals Phys. 324, 414 (2009) [arXiv:0805.2909 [hep-th]].

[10] D. Benedetti, P. F. Machado and F. Saueressig, Nucl. Phys. B 824, 168 (2010) [arXiv:0902.4630 [hep-th]].

[11] J. Ambjorn, J. Jurkiewicz and R. Loll, Phys. Rev. D 72, 064014 (2005) [hep-th/0505154].

[12] J. Ambjorn, A. Gorlich, J. Jurkiewicz and R. Loll, Phys. Rev. Lett. 100, 091304 (2008) [arXiv:0712.2485 [hep-th]].

[13] J. Ambjorn, A. Gorlich, J. Jurkiewicz and R. Loll, Phys. Rev. D 78, 063544 (2008) [arXiv:0807.4481 [hep-th]].

[14] H. W. Hamber, Gen. Rel. Grav. 41, 817 (2009) [arXiv:0901.0964 [gr-qc]].

[15] J. Ambjorn and J. Jurkiewicz, Phys. Lett. B 278, 42 (1992).

[16] M. E. Agishtein and A. A. Migdal, Mod. Phys. Lett. A 7, 1039 (1992).

[17] P. Bialas, Z. Burda, A. Krzywicki and B. Petersson, Nucl. Phys. B 472, 293 (1996) [hep-lat/9601024].

[18] B. V. de Bakker, Phys. Lett. B 389, 238 (1996) [hep-lat/9603024].

[19] B. V. de Bakker and J. Smit, Nucl. Phys. B 439, 239 (1995) [hep-lat/9407014].

[20] J. Ambjorn and J. Jurkiewicz, Nucl. Phys. B 451, 643 (1995) [hep-th/9503006].

[21] S. Catterall, J. B. Kogut and R. Renken, Phys. Lett. B 328, 277 (1994) [hep-lat/9401026].

[22] H. S. Egawa, T. Hotta, T. Izubuchi, N. Tsuda and T. Yukawa, Prog. Theor. Phys. 97, 539 (1997) [hep-lat/9611028].

[23] J. Ambjorn and R. Loll, Nucl. Phys. B 536, 407 (1998) [hep-th/9805108].

[24] J. Ambjorn, J. Jurkiewicz and R. Loll, Nucl. Phys. B 610, 347 (2001) [hep-th/0105267].

[25] J. Ambjorn, J. Jurkiewicz and R. Loll, Phys. Rev. Lett. 93, 131301 (2004) [hep-th/0404156].

[26] J. Ambjorn, J. Jurkiewicz and R. Loll, Phys. Lett. B 607, 205 (2005) [hep-th/0411152].

[27] J. Ambjorn, S. Jordan, J. Jurkiewicz and R. Loll, Phys. Rev. Lett. 107, 211303 (2011) [arXiv:1108.3932 [hep-th]].

[28] J. Ambjorn, J. Jurkiewicz and R. Loll, Phys. Rev. Lett. 95, 171301 (2005) [hep-th/0505113].

[29] J. Ambjorn, J. Jurkiewicz and R. Loll, Phys. Rev. D 72, 064014 (2005) [hep-th/0505154].

[30] O. Lauscher and M. Reuter, JHEP 0510, 050 (2005) [hep-th/0508202].

[31] P. Horava, Phys. Rev. Lett. 102, 161301 (2009) [arXiv:0902.3657 [hep-th]].

[32] D. Benedetti, Phys. Rev. Lett. 102, 111303 (2009) [arXiv:0811.1396 [hep-th]]. 
[33] J. Ambjorn, J. Jurkiewicz and R. Loll, PoS LATTICE 2010, 014 (2010) [arXiv:1105.5582 [hep-lat]].

[34] J. Ambjorn, A. Gorlich, J. Jurkiewicz and R. Loll, Phys. Lett. B 690, 420 (2010) [arXiv:1001.4581 [hep-th]].

[35] J. Laiho and D. Coumbe, Phys. Rev. Lett. 107, 161301 (2011) [arXiv:1104.5505 [hep-lat]].

[36] B. Bruegmann and E. Marinari, Phys. Rev. Lett. 70, 1908 (1993) [hep-lat/9210002].

[37] S. Bilke, Z. Burda, A. Krzywicki, B. Petersson, J. Tabaczek and G. Thorleifsson, Phys. Lett. B 432, 279 (1998) [hep-lat/9804011].

[38] J. Ambjorn, B. Durhuus and T. Jonsson, Cambridge, UK: Univ. Pr., 1997. (Cambridge Monographs in Mathematical Physics). $363 \mathrm{p}$

[39] M. Gross and S. Varsted, Nucl. Phys. B 378, 367 (1992).

[40] S. Bilke and G. Thorleifsson, Phys. Rev. D 59, 124008 (1999) [hep-lat/9810049].

[41] D. Coumbe and J. Laiho, PoS LATTICE 2011, 334 (2011) [arXiv:1201.2864 [hep-lat]].

[42] T. Jonsson and J. F. Wheater, Nucl. Phys. B 515, 549 (1998) [hep-lat/9710024].

[43] D. Coumbe and J. Laiho, In preparation.

[44] T. Banks, arXiv:1007.4001 [hep-th].

[45] A. Shomer, arXiv:0709.3555 [hep-th].

[46] R. Percacci and G. P. Vacca, Class. Quant. Grav. 27, 245026 (2010) [arXiv:1008.3621 [hep-th]].

[47] E. Akkermans, G. V. Dunne and A. Teplyaev, Phys. Rev. Lett. 105, 230407 (2010) [arXiv:1010.1148 [cond-mat.stat-mech]].

[48] O. J. Rosten, arXiv:1106.2544 [hep-th].

[49] M. Reuter and F. Saueressig, JHEP 1112, 012 (2011) [arXiv:1110.5224 [hep-th]].

[50] J. Ambjorn, A. Goerlich, J. Jurkiewicz and R. Loll, arXiv:1203.3591 [hep-th].

[51] H. W. Hamber, Phys. Rev. D 45, 507 (1992).

[52] S. Catterall, D. Ferrante and A. Nicholson, arXiv:0912.5525 [hep-lat].

[53] M. Shaposhnikov and C. Wetterich, Phys. Lett. B 683, 196 (2010) [arXiv:0912.0208 [hep-th]]. 